\title{
A SELF-CENTRALIZING CHARACTERISTIC SUBGROUP
}

\author{
M. D. PEREZ-RAMOS
}

(Received 24 April 1987)

Communicated by H. Lausch

\begin{abstract}
In this note we introduce a self-centralizing characteristic subgroup, associated with quasinilpotent injectors, of a finite group.

1980 Mathematics subject classification (Amer. Math. Soc.) (1985 Revision): 20 D 10.
\end{abstract}

All groups in this paper are assumed to be finite. Most of our notation is standard and may be found in [9] and [10]. Let $d(G), d_{\infty}(G)$ denote, respectively, the maximum of the orders of the abelian and nilpotent subgroups of $G$. Further, $\mathbf{A}(G)\left[\mathbf{A}_{\infty}(G)\right]$ denotes the set of all abelian [nilpotent] subgroups of order $d(G)$ $\left[d_{\infty}(G)\right]$ in $G$ and $J(G)=\langle\mathbf{A}(G)\rangle$ is the Thompson subgroup of $G$.

Let $p$ be a prime and let $P$ be a Sylow $p$-subgroup of a group $G$. In [8] Glauberman introduces the characteristic subgroup $Z J^{*}(P)$, and proves that $Z J^{*}(P)$ has some analogous properties to $Z J(P)=Z(J(P))$, and moreover that it is self-centralizing, that is, $C_{G}\left(Z J^{*}(P)\right) \leq Z J^{*}(P)$. Some related results were obtained by Ezquerro in [6], where Glauberman's definition of $Z J^{*}$ is set forth for an arbitrary finite group $G$ (instead of $P$ ).

DEFINITIONS. For any group $K$ define two sequences of characteristic subgroups of $K$ as follows. Let $Z J^{0}(K)=1$ and $K_{0}=K$. Given $Z J^{i}(K)$ and $K_{i}$, $i \geq 0$, let $Z J^{i+1}(K)$ and $K_{i+1}$ be the subgroups of $K$ that contain $Z J^{i}(K)$ and

(C) 1989 Australian Mathematical Society $0263-6115 / 89 \$ A 2.00+0.00$ 
satisfy

$$
\begin{gathered}
Z J^{i+1}(K) / Z J^{i}(K)=Z J\left(K_{i} / Z J^{i}(K)\right) \\
K_{i+1} / Z J^{i}(K)=C_{K_{i} / Z J^{i}(K)}\left(Z J^{i+1}(K) / Z J^{i}(K)\right) .
\end{gathered}
$$

Let $n$ be the smallest integer such that $Z J^{n}(K)=Z J^{n+1}(K)$; then $Z J^{n}(K)$ $=Z J^{n+r}(K)$ and $K_{n}=K_{n+r}$, for every $r \geq 0$. Set $Z J^{*}(K)=Z J^{n}(K)$ and $K_{*}=K_{n}$.

The main aim of this paper is to prove the following

THEOREM. Let $G$ be a group such that for every odd $p$ in $\Pi(G / Z(G))$, the special affine group $S A(2, p)$ is not involved in $G$. Let $K$ be an $\mathrm{N}^{*}$-injector of $G$ and assume that $|F(G)| \neq 1$ is odd. Then

(i) $Z J^{i}(K)$ char $G$, for every $i \geq 0$; in particular, $Z J^{*}(K) \operatorname{char} G$;

(ii) $C_{G}\left(K_{*}\right) \leq K_{*}=E(G) Z J^{*}(K)$; indeed, $C_{G}\left(K_{*}\right)=Z\left(Z J^{*}(K)\right)$.

Here $\Pi$ denotes a set of primes and $\mathbf{N}, \mathbf{N}_{\Pi}$ and $\mathbf{N}^{*}$ denote the classes of nilpotent, nilpotent $\Pi$-groups and quasinilpotent groups, respectively. It is well known that all groups have a unique conjugacy class of $\mathbf{N}^{*}$-injectors, and these are the maximal $\mathbf{N}^{*}$-subgroups containing the quasinilpotent radical of the group [5].

The following simple observations are due to L. M. Ezquerro [6].

LEMMA 1. (i) If $\psi: K \rightarrow H$ is an isomorphism, then for every $i \geq 0$, $Z J^{i}(H)=\psi\left(Z J^{i}(K)\right)$.

(ii) $Z J^{i+1}(K) / Z J^{1}(K)=Z J^{i}\left(K_{1} / Z J^{1}(K)\right)$ for every $i \geq 0$.

(iii) $C_{K}\left(Z J^{*}(K)\right) \leq K_{i}$ for every $i \geq 0$.

LEMMA 2. Let $K$ be an $\mathbf{N}^{*}$-injector of the group $G$.

(i) For every $i \geq 0, Z J^{i}(K)$ is nilpotent.

(ii) $K_{i}=E(G) F\left(K_{i}\right)$ and $F\left(K_{i} / Z J^{i}(K)\right)=F\left(K_{i}\right) / Z J^{i}(K)$, for every $i \geq 0$.

(iii) $Z J^{i+1}(K)=Z J^{i}(K)$ if and only if $F\left(K_{i}\right)=Z J^{i}(K)$. Thus, $K_{*}=$ $E(G) Z J^{*}(K)$.

ProOF. (i) By induction on $i$ we may assume that for some $i, Z J^{i}(K)$ is nilpotent. Then $Z J^{i+1}(K) \in N^{*}$ is soluble and hence nilpotent.

(ii) Notice that for every $i \geq 0, E(G) \leq K_{i} \unlhd K=E(G) F(K)$, so $K_{i}=$ $E(G) F\left(K_{i}\right)$. For each $i \geq 0$, put $F / Z J^{i}(K)=F\left(K_{i} / Z J^{i}(K)\right)$. Then, since $Z J^{i}(K) \in \mathrm{N}, F \in \mathrm{N}^{*}$ is soluble. Consequently, $F$ is nilpotent and $F\left(K_{i} / Z J^{i}(K)\right)$ $=F\left(K_{i}\right) / Z J^{i}(K)$. 
(iii) For each $i \geq 0$, we obtained from (ii) that

$$
\begin{aligned}
K_{i} / Z J^{i}(K) & =\left(E(G) Z J^{i}(K) / Z J^{i}(K)\right)\left(F\left(K_{i}\right) / Z J^{i}(K)\right) \\
& =\left(E(G) Z J^{i}(K) / Z J^{i}(K)\right)\left(F\left(K_{i} / Z J^{i}(K)\right)\right) .
\end{aligned}
$$

Thus our claim is clear from $Z J\left(K_{i} / Z J^{i}(K)\right) \neq 1 \leftrightarrow F\left(K_{i} / Z J^{i}(K)\right) \neq 1$.

Definition [14, Definition 1]. Let $\mathbf{F}$ be a Fitting class. A group $G$ is said to be F-stable if whenever $A$ is an F-subgroup of $G$ and $B$ is an F-subgroup of $N_{G}(A)$ such that $[A, B, B]=1$, then $B C_{G}(A) / C_{G}(A) \leq\left(N_{G}(A) / C_{G}(A)\right)_{\mathrm{F}}$.

LEMMA $3[15]$. The class $\mathbf{N}_{\Pi}^{*}=(G=F(G) E(G)$ such that $F(G)$ is a $\Pi$ group) is a Fitting class.

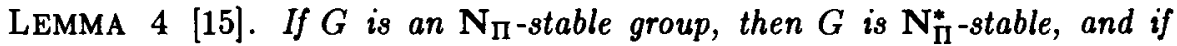
$A$ and $B$ are $\mathrm{N}_{\Pi}^{*}$-subgroups such that $B$ normalizes $A$ and $[A, B, B]=1$, then $B C_{G}(A) / C_{G}(A) \leq O_{\Pi}\left(F\left(N_{G}(A) / C_{G}(A)\right)\right)$.

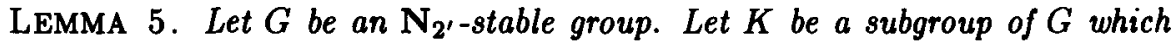
contains $F^{*}(G)$. Then $O_{2^{\prime}}(Z J(K)) \triangleleft \triangleleft G$. Moreover, if $|F(G)|$ is odd, then $Z J(K) \triangleleft \triangleleft G$.

PROOF. Using Lemma 4 on $F=E(G) O_{2^{\prime}}(F(G))$ and $Z=O_{2^{\prime}}(Z J(K))$ we obtain $Z C_{G}(F) / C_{G}(F) \leq F\left(G / C_{G}(F)\right)$. Hence $Z C_{G}\left(F^{*}(G)\right)=Z C_{G}(F) \cap$ $C_{G}\left(O_{2}(F(G))\right) \triangleleft \triangleleft G$. Since $C_{G}\left(F^{*}(G)\right) \leq F^{*}(G), Z \triangleleft \triangleleft G$. Moreover if $|F(G)|$ is odd, then $O_{2}(Z J(K)) \leq C_{G}\left(F^{*}(G)\right) \leq F(G)$ and $O_{2}(Z J(K))=1$.

Now, arguing like in the proof of $[14$, Theorem A], with small changes, we obtain

Proposition 1. Let $G$ be an $\mathbf{N}_{2}$-stable group such that $F(G) \neq 1$ is not a 2-group. If $K$ is an $\mathrm{N}^{*}$-injector of $G$, then $1 \neq O_{2^{\prime}}(Z J(K)) \triangleleft G$. In particular if $|F(G)|$ is odd, then $Z J(K) \triangleleft G$.

ProOF OF THE THEOREM. (i) Assume that the result is false. Let $G$ be counterexample of least order and put $T=Z J(K), T^{i}=Z J^{i}(K), T^{*}=$ $Z J^{*}(K)$. By [7, Theorem A] $G$ is $\mathrm{N}_{2^{\prime}}$-stable. Therefore, because of Proposition 1 we have $1 \neq T \triangleleft G$. Thus, $C=C_{G}(T) \neq 1$. Assume that $C<G$.

By our minimal choice of $G$, we have that for each $i \geq 0, Z J^{i}(K \cap C) \operatorname{char} C$. Since $J(K) \leq K \cap C$, it follows that $J(K)=J(K \cap C)$ and $T=Z J(K \cap C)$. Moreover, $K_{1}=C_{K}(T)=C_{K \cap C}(Z J(K \cap C))$.

By induction on $i$, it is clear that for each $i \geq 0, T^{i}=Z J^{i}(K \cap C)$. Thus, for every $i \geq 0, T^{i} \unlhd G$. But Lemma 1 (i) and the conjugacy of all $\mathbf{N}^{*}$-injectors of 
$G$ imply that for every $i \geq 0, T^{i} \operatorname{char} G$, contrary to our choice of $G$. Therefore we can assume that $G=C$, (that is, $Z(G)=T$ ), and that $G / Z(G)$ satisfies the hypothesis of the theorem. By our minimal choice of $G$, we obtain that for every $i \geq 0, Z J^{i}(K / Z(G))$ char $G / Z(G)$. Since $K_{1}=C_{K}(T)=K$, and by Lemma 1 (ii), it follows that for each $i \geq 0, T^{i+1} / Z(G)=Z J^{i}(K / Z(G))$. Thus for every $i \geq 0, T^{i+1}$ char $G$, contrary to our choice of $G$.

(ii) By Lemma 2 (iii) we know that $K_{*}=E(G) T^{*}$. Now, in view of Lemma 1 (iii), it is clear that for every $i \geq 0, C_{K}\left(K_{*}\right) \leq C_{K}\left(T^{*}\right) \leq K_{i}$. In particular, $C_{K}\left(K_{*}\right) \leq K_{*}$. Thus, $C_{K}\left(K_{*}\right)=Z\left(K_{*}\right)=Z\left(T^{*}\right)$.

Part (i) and Lemma 2 (iii) imply that $C_{G}\left(K_{*}\right) \unlhd G$. Hence $Z\left(K_{*}\right)=K \cap$ $C_{G}\left(K_{*}\right)$ in an $\mathrm{N}^{*}$-injector of $C_{G}\left(K_{*}\right)$. Moreover, since $Z\left(K_{*}\right) \leq Z\left(C_{G}\left(K_{*}\right)\right)$, we get that $Z\left(K_{*}\right)=Z\left(C_{G}\left(K_{*}\right)\right)=F\left(C_{G}\left(K_{*}\right)\right)$. But from $C_{G}\left(K_{*}\right) \unlhd C_{G}(E(G))$ we get that $C_{G}\left(K_{*}\right)$ is $\mathrm{N}$-constrained in the sense of [12]; see [11], [13]. Therefore, $C_{G}\left(K_{*}\right)=C_{C_{G}\left(K_{*}\right)}\left(Z\left(K_{*}\right)\right)=Z\left(K_{*}\right)$.

If $G$ is an $\mathrm{N}$-constrained group, then $E(G)=1$ and the $\mathbf{N}$-injectors are the $\mathrm{N}^{*}$-injectors (see [12], [13] and [11]). So, we obtain immediately

COROLLARY 1. Under the same assumptions as in the theorem and if moreover $G$ is $N$-constrained, then $K$ is an $\mathrm{N}$-injector of $G, Z J^{*}(K) \operatorname{char} G$ and

$$
C_{G}\left(Z J^{*}(K)\right) \leq Z J^{*}(K) \text {. }
$$

Next we shall consider an analogue of our Theorem for arbitrary Fitting classes F.

Proposition 2 [6, TheOREM II.3.10]. Assume that $2 \notin \Pi$ and $G$ is a $\Pi$ soluble group whose Sylow 2-subgroups are abelian and such that $O_{\Pi^{\prime}}(G)=1$. If $K$ is a Hall $\Pi$-subgroup of $G$, then $Z J^{*}(K) \operatorname{char} G$ and $C_{G}\left(Z J^{*}(K)\right) \leq Z J^{*}(K)$.

LEMMA 6. Assume that $2 \notin \Pi$ and $G$ is a $\Pi$-soluble group and $H$ is a subgroup of $G$ contained in a Hall $\Pi$-subgroup $K$ of $G$ such that $d_{\infty}(H)=d_{\infty}(K)$. Let $A \in \mathbf{A}_{\infty}(H)$. Then $Z J^{i}(A)=Z J^{i}(H)=Z J^{i}(K)$, for every $i \geq 0$. In particular, $Z J(H)=Z J(K)$ and $Z J^{*}(H)=Z J^{*}(K)$.

Proof. Notice first that if $G$ is a group of odd order, then $\mathbf{A}_{\infty}(G)$ is the set of $\mathrm{N}$-injectors of $G$. (See [4, Corollary 5$]$ and [12, Theorem 1].)

We proceed by induction on $i$. Because of [3, Proposition (1.3)], it follows that $Z J(H)=Z J(A)=Z J(K)$. Assume now that for some $i \geq 1, Z J^{i}(H)=$ $Z J^{i}(A)=Z J^{i}(K)$. Set $Z=Z J(A)=Z J(H)=Z J(K)$. Note that $A_{1}=$ $C_{A}(Z)=A \cap H_{1}=A \cap K_{1}$ is an N-injector of $H_{1}=C_{H}(Z)$ and $K_{1}=C_{K}(Z)$, 
and obviously $Z \leq A_{1}$. Hence, from [12, Theorem 1] we get that $A_{1} / Z$ is an $\mathrm{N}$-injector of $H_{1} / Z$ and of $K_{1} / Z$. Therefore, $A_{1} / Z \in \mathbf{A}_{\infty}\left(H_{1} / Z\right) \subseteq \mathbf{A}_{\infty}\left(K_{1} / Z\right)$.

The group $C_{G}(Z) / Z$ satisfies the hypothesis concerning $G$. Thus,

$$
Z J^{i}\left(A_{1} / Z\right)=Z J^{i}\left(H_{1} / Z\right)=Z J^{i}\left(K_{1} / Z\right) \text {. }
$$

Now, from Lemma 1 (ii) we have

$$
Z J^{i+1}(A)=Z J^{i+1}(H)=Z J^{i+1}(K) .
$$

In what follows let $\Pi(F)$ denote the set of all primes dividing the order of some F-group. The next lemma and proposition are proved following a process analogous to the proofs of Lemma IV.2.3 and Theorem IV.2.4 in [13]. We denote by $\mathbf{F}$ a Fitting class.

LEMMA 7. Let $G$ be a $\Pi(\mathbf{F})$-soluble group and $N \unlhd G$ such that $G / N \in \mathbf{N}$. If $V_{1}$ and $V_{2}$ are $\mathbf{F}$-maximal subgroups of $G$ which contain an $\mathbf{F}$-maximal subgroup $W$ of $N$, then there exists $g \in G$ such that $V_{1}^{g}=V_{2}$.

Proposition 3. If $G$ is a $\Pi(\mathbf{F})$-soluble group, then $G$ has a unique conjugacy class of F-injectors.

REMARK. If $2 \notin \Pi=\Pi(\mathbf{F})$ and $H$ is an $\mathbf{F}$-injector of the $\Pi$-soluble group $G$ contained in the Hall $\Pi$-subgroup $K$ of $G$ such that $d_{\infty}(H)=d_{\infty}(K)$, then by Lemma 6, for every $i \geq 0$ and $A \in \mathbf{A}_{\infty}(H)$,

$$
Z J^{i}(A)=Z J^{i}(H)=Z J^{i}(K)
$$

and in particular, $Z J(H)=Z J(K)$ and $Z J^{*}(H)=Z J^{*}(K)$.

From Proposition 2 and the preceding remark we obtain the following

COROLLARY 2. Assume that $2 \notin \Pi=\Pi(\mathbf{F})$ and $G$ is a $\Pi$-soluble group with abelian Sylow 2-subgroups and $O_{\Pi^{\prime}}(G)=1$. If $H$ is an $\mathrm{F}$-injector of $G$ contained in the Hall $\Pi$-subgroup $K$ of $G$ such that $d_{\infty}(H)=d_{\infty}(K)$, then $Z J^{*}(H) \operatorname{char} G$ and $C_{G}\left(Z J^{*}(H)\right) \leq Z J^{*}(H)$.

\section{Acknowledgements}

This note is part of the author's Doctoral Thesis at the University of Valencia, Spain. The author wishes to express her gratitude to her supervisor Dr. Perez Monasor and also to Dr. Iranzo for their devoted guidance and encouragement. The author was supported by a scholarship from the "Ministerio de Educacion y Ciencia" of Spain. 


\section{References}

[1] Z. Arad, ' $\Pi$ '-closure of finite П-solvable groups', J. Austral. Math. Soc. Ser. A. 21 (1976), 118-119.

[2] Z. Arad, 'Abelian and nilpotent subgroups of maximal order of groups of odd order', Pacific J. Math. 62 (1976), 29-35.

[3] Z. Arad and D. Chillag, 'Injectors of finite solvable groups', Comm. Algebra 7 (1979), 115-138.

[4] A. Bialostocki, 'On products of two nilpotent subgroups of a finite group', Israel J. Math. 20 (1975), 178-188.

[5] D. Blessenohl and H. Laue, 'Fittingklassen endlicher Gruppen, in denen gewise Hauptfaktoren einfach sind', J. Algebra 56 (1979), 516-532.

[6] L. M. Ezquerro, 'F-estabilidad, constriccion y factorizacion de grupos finitos', Tesis Doctoral, U. de Valencia (1983).

[7] L. M. Ezquerro, 'F-stability of finite groups', J. Algebra 92 (1985), 395-399.

[8] G. Glauberman, 'A characteristic subgroup of a p-stable group', Canad. J. Math. 20 (1968), 1101-1135.

[9] D. Gorenstein, Finite groups (Harper \& Row, New York, 1968).

[10] B. Huppert and N. Blackburn, Finite groups, II, III (Springer-Verlag, Berlin, 1982).

[11] M. J. Iranzo and F. Perez Monasor, 'Existence of $\mathbf{N}$-injectors in a not central normal Fitting class', Israel J. Math. 48 (1984), 123-128.

[12] A. Mann, 'Injectors and normal subgroups of finite groups', Israel J. Math. 9 (1971), 554-558.

[13] F. Perez Monasor, 'Grupos finitos separados respecto de una formacion de Fitting', Rev. Acad. Ciencias de Zaragoza, Serie 228 (1973).

[14] M. D. Perez-Ramos, 'A characteristic subgroup of N-stable groups', Israel J. Math. 54 (1986), 51-59.

[15] M. D. Perez-Ramos, 'Stability with respect to a Fitting class', J. Algebra, to appear.

Departamento de Algebra

Universidad de Valencia

C/Doctor Moliner, 50

46100 Burjassot

Valencia, Spain 